\title{
Lethargy in a patient with diabetes
}

\author{
A diagnosis not to be missed
}

Kosar Hussain, Sara Hussain, Muhammad Hassan

\section{CASE}

A man aged 61 years presented with a two-week history of epigastric discomfort, nausea and lethargy. He did not have any infective symptoms, and there was no history of any recent illness. He had known type 2 diabetes, which had been diagnosed 12 years ago. He had no evidence of significant microvascular or macrovascular complications of diabetes. Insulinoma antigen-2 (IA2) and glutamic acid decarboxylase (GAD) antibody levels were not elevated. His glycated haemoglobin level generally ranged between $58 \mathrm{mmol} / \mathrm{mol}$ and $64 \mathrm{mmol} / \mathrm{mol}$ (7.5\% and 8.0\%).

His diabetic medications included metformin 2 g daily, dapagliflozin $10 \mathrm{mg}$ daily, insulin glargine 12 units at night and insulin aspart as per a sliding scale ranging from four to 12 units three times daily. He had been on the same regimen for the past two years. He took all his medications regularly and reported his blood sugar levels for the last few days were between $6 \mathrm{mEq} / \mathrm{L}$ and $14 \mathrm{mEq} / \mathrm{L}$. He was an ex-smoker and reported having 1-2 units of alcohol daily.

On initial examination, the patient appeared well and had normal vital signs. General examination was unremarkable. His initial blood sugar level was $12.9 \mathrm{mmol} / \mathrm{L}$, and urinary ketones were strongly positive. The patient was referred to the emergency department for further assessment. Blood gas results showed a $\mathrm{pH}$ of 7.17, partial pressure of carbon dioxide of $40 \mathrm{mmHg}$, bicarbonate concentration of $14 \mathrm{mEq} / \mathrm{L}$, anion gap of 26 , base excess of $-13 \mathrm{mmol} / \mathrm{L}$ and normal lactate levels. The serum ketone level was elevated at $6.0 \mathrm{mmol} / \mathrm{L}$. The result of septic work-up was negative. The remaining blood investigations including lipase, cardiac enzymes and renal and liver function were all within normal limits. Electrocardiography showed a normal sinus rhythm.

\section{QUESTION 1}

What is the differential diagnosis of euglycaemic ketoacidosis?

\section{ANSWER 1}

The differential diagnosis of euglycaemic ketoacidosis includes:

- fasting ketoacidosis - occurs after periods of prolonged fasting in infants, young children or pregnant women ${ }^{1}$

- alcoholic ketoacidosis - classically presents in patients with a history of chronic alcohol abuse and malnutrition, after a recent episode of binge drinking

- euglycaemic diabetic ketoacidosis (euDKA) - occurs in patients with poor oral intake, those who pre-treat with insulin prior to arrival at the emergency department, pregnant women and people taking sodium-glucose co-transporter-2 (SGLT2) inhibitors..$^{2,3}$

\section{CASE CONTINUED}

The patient had presented with high anion gap metabolic acidosis that required hospitalisation. His presentation was typical of diabetic ketoacidosis (DKA), yet the blood sugar levels were not convincingly high. He had no previous history of DKA in the past, and there were no other precipitating factors to explain the occurrence of ketosis, other than empagliflozin. euDKA secondary to empagliflozin was suspected. Empagliflozin was withheld, and the patient was treated with insulin and dextrose infusion. The ketoacidosis resolved in 24 hours, and the patient was transitioned back to subcutaneous insulin. He was discharged home after three days in good health, after correction of ketosis.

\section{QUESTION 2}

Which subset of patients with diabetes would benefit from the addition of SGLT2 inhibitors to their regimen?

\section{QUESTION 3}

What are the risk factors for developing euDKA among patients receiving SGLT2 inhibitors?

\section{QUESTION 4}

What is the pathophysiology of euDKA secondary to SGLT2 inhibitors?

\section{QUESTION 5}

What is the incidence of euDKA secondary to SGLT2 inhibitors? 


\section{QUESTION 6}

What pertinent precautions must be practised for patients taking SGLT2 inhibitors?

\section{ANSWER 2}

SGLT2 inhibitors are novel oral medications that act by preventing glucose reabsorption in the renal tubule, thus promoting glucosuria. ${ }^{4}$ They are listed on the Pharmaceutical Benefits Scheme as a second- and third-line option for patients with type 2 diabetes who have not achieved glycaemic targets with metformin and/or a sulfonylurea.

\section{ANSWER 3}

Commonly, there are obvious triggering factors such as an intercurrent illness, surgery, reduced food and fluid intake, reduced insulin doses, previous ketoacidosis, a missed diagnosis of type 1 diabetes or latent autoimmune diabetes of adulthood, and history of alcohol abuse. ${ }^{5}$ At other times, such as in this case, there is no clear triggering factor to explain the occurrence of euDKA.

There are certain important considerations before starting SGLT2 inhibitors: ${ }^{6}$

- Dapagliflozin should be avoided for patients with moderate-tosevere renal impairment (estimated glomerular filtration rate [eGFR] $\left.<60 \mathrm{~mL} / \mathrm{min} / 1.73 \mathrm{~m}^{2}\right)$. It is also not recommended with concomitant loop diuretic use and for patients aged $\geq 75$ years because of increased risk of volume depletion.

- Empagliflozin should be avoided in patients with severe renal impairment $\left(\mathrm{eGFR}<30 \mathrm{~mL} / \mathrm{min} / 1.73 \mathrm{~m}^{2}\right)$. It is not recommended with concomitant loop diuretic use and for patients aged $\geq 85$ years.

\section{ANSWER 4}

Because insulin deficiency and resistance are milder in euDKA, glucose overproduction and underutilisation are quantitatively lower than in DKA. More importantly, renal glucose clearance is much higher with euDKA than with DKA. Subsequently, the degree of hyperglycaemia is much milder in euDKA. ${ }^{7}$
In fact, it is the absence of significant hyperglycaemia that precludes the recognition of this significant complication by both patients and medical practitioners.

\section{ANSWER 5}

Based on a total of 85,851 dapagliflozin and empagliflozin prescriptions written in South Australia from December 2015 to March 2017, there were 13 identified cases of eDKA. This equates to an event rate of 1.8 cases per 1000 patient-years. ${ }^{5}$ However, the majority of these adverse effects are generally under-reported. ${ }^{8}$

\section{ANSWER 6}

euDKA secondary to SGLT2 inhibitors can result in severe and life-threatening acidosis requiring admission to a high-dependency unit or intensive care unit for supportive care.

Patients must be educated about the signs and symptoms of ketoacidosis and be instructed to seek immediate medical attention if those symptoms appear.

SGLT2 inhibitors must be withheld during acute illness and prior to operations.

Serum or urine ketones should be monitored for any patient with nausea, lethargy or abdominal pain.

Routine monitoring of both blood glucose and serum/urine ketone levels is important if the patient appears unwell.

\section{Key points}

- Routine monitoring of both blood glucose and serum/urine ketone levels is important in any patient taking SGLT2 inhibitors who presents with nausea, lethargy or abdominal pain.

- A diagnosis of euDKA should be considered for patients taking SGLT2 inhibitors who present with elevated ketones and normal blood sugar levels.

- SGLT2 inhibitors should be discontinued indefinitely if acidosis is confirmed, as the subgroup of patients treated with SGLT2 could have ketosis-prone type 2 diabetes.
Sara Hussain MBBS, Resident, Emergency Department, Rashid Hospital, Dubai; Dubai Health Authority, Dubai, United Arab Emirates

Muhammad Hassan MBBS, GP Registrar, Tatura Medical Centre, Vic

Competing interests: None.

Funding: None.

Provenance and peer review: Not commissioned, externally peer reviewed.

\section{References}

1. Munro JF, Campbell IW, McCuish AC, Duncan LJ. Euglycaemic diabetic ketoacidosis. Br Med J 1973;2(5866):578-80. doi: 10.1136/bmj.2.5866.578.

2. Qiu H, Novikov A, Vallon V. Ketosis and diabetic ketoacidosis in response to SGLT2 inhibitors: Basic mechanisms and therapeutic perspectives. Diabetes Metab Res Rev 2017;33(5):e2886. doi: 10.1002/dmrr.2886.

3. Fralick M, Schneeweiss S, Patorno E. Risk of Diabetic ketoacidosis after initiation of an SGLT2 inhibitor. N Engl J Med 2017;376(23):2300-02. doi: 10.1056/NEJMc1701990.

4. Tahrani AA, Barnett AH, Bailey CJ. SGLT inhibitors in management of diabetes. Lancet Diabetes Endocrinol 2013;1(2):140-51. doi: 10.1016/S22138587(13)70050-0.

5. Meyer EJ, Gabb G, Jesudason D. SGLT2 inhibitor-associated euglycemic diabetic ketoacidosis: A South Australian clinical case series and Australian spontaneous adverse event notifications. Diabetes Care 2018;41(4):e47-e9. doi: 10.2337/dc17-1721.

6. NPS MedicineWise. RADAR SGLT2 inhibitor listings: Indications and combinations. Surrey Hills, NSW: NPS MedicineWise, 2015. Available at www.nps.org.au/radar/articles/sglt2-inhibitorlistings-indications-and-combinations [Accessed 12 July 2019].

7. Rosenstock J, Ferrannini E. Euglycemic diabetic ketoacidosis: A predictable, detectable, and preventable safety concern with SGLT2 inhibitors. Diabetes Care 2015;38(9):1638-642. doi: 10.2337/ dc15-1380.

8. Hazell L, Shakir SA. Under-reporting of adverse drug reactions: A systematic review. Drug Saf 2006;29(5):385-96.

\section{Authors}

Kosar Hussain MBBS, Medical Registrar, General Medicine, Goulburn Valley Health, Vic dr.kosar.hussain@gmail.com 